\title{
Development of engineering approach to the assessment of stress raisers in metal structures of dragline excavators
}

\author{
Petr Pobegailo ${ }^{1}$, Dmitry Kritsky ${ }^{2}$, Nikolai Suslov ${ }^{3}$, and Tatiana Gilmanshina ${ }^{4}$ \\ ${ }^{1}$ IMASH named after A.A. Blagonravov RAS, 101000, Moscow, Russian Federation; \\ ${ }^{2}$ SUEK-KRASNOYARSK JSC, 660049, Krasnoyarsk, Russian Federation \\ ${ }^{3}$ FSBOU "Ural State Mining University", 620144, Yekaterinburg, Russian Federation \\ ${ }^{4}$ FSAOU "Siberian Federal University", 660041, Krasnoyarsk, Russian Federation
}

\begin{abstract}
In the present work, its authors present the new results obtained on the creation and development of an engineering approach to the assessment of stress raisers in the metal structures of dragline excavators, convenient to be applied in the express diagnostics system to test the condition of such machines. The main result of this work is the conclusion that the value of the geometric stress concentration factor for the fillet weld of metal structures of the excavator arm EH-10/70A under working conditions can be written using the Afanasiev formula.
\end{abstract}

\section{Introduction}

The principal characteristic feature of a single-bucket dragline excavator is the presence of a large number of welded metal structures in their design. It is associated with the presence of local areas with increased stress factor in them, and the main place where stress factors occur happens to be the welds. Such welds are significantly subjected to fatigue damage (especially since they are subjected to alternate load when dragline is operated).

Generally speaking, the stress factor can be characterized by a geometric stress concentration factor [ 1 and others] (as well as by an effective stress concentration factor that we will no longer mention in this article).

The ratio of the maximum stress in the stress concentration region to the nominal stress of the part, the strain being elastic, assuming that the material meets the conditions of a continuous homogeneous medium happens to be a geometric stress concentration factor (TKK) $\alpha_{\sigma}[1$, and many others].

Nearly a hundred years ago, there first appeared papers to study various issues related to stress concentration. The following scientists carried out this research: Afanasiev N.N., Bakshi O.A., Belchuk G.A., Vinokrov V.A., Danilov S.A., Zaytsev N.A., Karnakhin V.A., Karkhin V.V., Klykov V.A., Kogayev V.P., Kopelman L.A., Leikin A.S., Makarov I.I., Muskhelishvili N.N., Navrotsky D.I., Neiber G., Nikolaev G.A., Oding I.A., Okumura T., Popkovich P.F., Peterson R., Prokofieva-Mikhailovskaia L. E., Ribin Yu.I., Savin G.N., Serenko A.N., Solokian A., Trufyakov V.I., Turmov G.P., Forrester P., Harikava K., 
Shimanskiy Yu.A., Schneiderovich R.M., Shron L.B., Endo Isira, and others. Ryakhin V.A. and his students made a certain contribution to these studies in the sphere of road construction machinery.

Various expressions for determining TKK stress have been proposed in the papers of these authors. All of these formulas are approximate, contain many different hypotheses and can be applied within sufficiently narrow limits. However, there are usually several formulas for the same weld type, and it is not always clear enough which of them is the best for a particular case.

At the same time, it should be noted that at present the operating company cannot rely upon the method of combined finite strip-finite elements, up-to-date computers and the involvement of scientists from the Academy of Sciences into the process of research. The issues of assessment of the condition of bearing metal structures of dragline excavators should be solved right away and in a very fast way even if the results are rather approximate. There is an acute need for automated methods of express diagnostics connected with nondestructive inspection technique.

Therefore, the main purpose of the papers planned by the authors is to determine under what conditions and in what range a given formula of TKK can be applied; which formula is more effective and more accurate for mining machinery, in particular, for draglines. In parallel, this will involve the development of appropriate software and the synthesis of a scientifically sound methodology for the selection and application of nondestructive inspection technique.

\section{Materials and methods}

\subsection{Theory}

In this small-scale work the authors present only some of the results they have obtained, and they have dealt only with fillet welds. The authors took the dragline excavator EH$10 / 70 \mathrm{~A}$ as an example due to the fact that this type of excavator predominated in the USSR, which gave an opportunity to one of the authors to get the necessary basic data to carry out the research.

It should be noted that during the formation of basic data we considered welded joints of the boom metal structure, in which the appearance of cracks was observed. Geometric sizing of welds was carried out according to the standard procedure [1 and others].

It should be emphasized that in this very paper the authors consider only two possible formulas for the estimation of the TKK: the formula of Afanasiev (1) and the formula of Nikolayev (2). They are as follows [1 - 3 and others]:

$$
\alpha_{\sigma}=1,6-0,2 \cdot \alpha+\frac{1}{7 \cdot \sqrt{\alpha^{3}} \cdot\left(1+\frac{1}{\varphi}\right)+0,4 \cdot \sqrt[3]{\alpha}}
$$

and

$$
\alpha_{\sigma}=1+0,18 \cdot\left(\frac{\delta}{\rho}\right)^{0,66}
$$


where $\alpha=\frac{\rho}{\delta}$ and $\varphi=\frac{t}{\delta}$ and, in turn: $\delta$ _ thickness of the welded metal; $\rho_{\text {. }}$ transition radius; $t$ - thickness of base metal.

Obviously, the TKK values are estimated for each section of the cast removed from a given weld pattern (because each weld section is characterized by its own size dimension). As a result, there is some random distribution of TKK requiring statistical processing. At the same time we have a small sample size ( $N<50$ ).

Referring to the works $[4,5$, etc.] we now estimate the following:

- sample average value - $\bar{m}_{\alpha_{\sigma}}$;

- sampling variance - $D^{2}$;

- sample mean square deviation - $D$;

- sample mean coefficient of variation $v_{-}$;

- confidence limits;

- value of cumulative frequency;

- parameters of created function graph on the normal probability grid;

- confidence limits for quantiles;

- intensity of errors related to the volume of available and required sampling.

It should be noted that the calculation of sample moments of the third and fourth order at our sample size is impractical $[4,5$, etc.]. At the same time, under the assumption of the normal law of distribution in our task, the estimates we have obtained are wealthy, effective and bias-free.

\subsection{Example}

By way of illustration of the carried out research, we will now focus at only one fillet weld on the support joint plate in the upper section of the lower boom belt (manual welding). Sample size is forty-nine (small sample). We have obtained the same results that for the other similar welds.

Thus, Figure 1 shows the behavior of TKK calculated by formula (1). The figure with the behavior of TKK calculated by formula (2) is not given now.

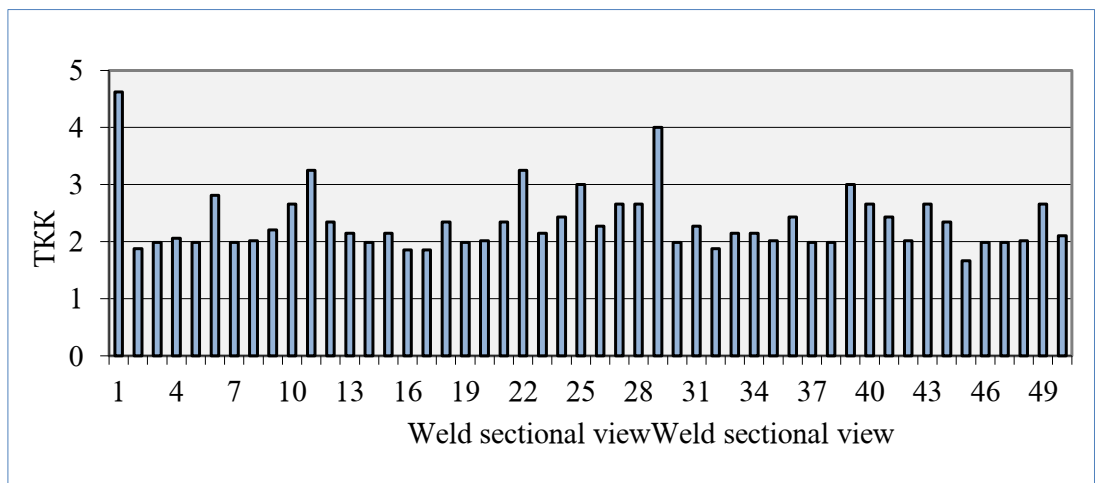

Fig. 1. Behavior of TKK obtained by formula (1) 
Thus, the TKK value estimated by formula (1) is in the range of 1,664 to 4,620 , and by formula (2) is in the range of 1,284 to 2,300 for the fillet weld under research.

In such a case, the obtained by formula (1) TKK are characterized by the following statistical estimation: $\bar{m}_{\alpha_{\sigma}}=2,346$ and $D^{2}=0,302, D=0,550$ and $v=0,234$. But the TKK value estimated by formula (2) are characterized by the following: $\bar{m}_{\alpha_{\sigma}}=1,582$ and $D^{2}=0,036, D=0,190$ and $v=0,120$

The $90 \%$ confidential interval for the universal mean at indeterminate variance at known sampling characteristics for a number of data obtained by formula (1) is as follows: $2,217<a<2,475$ and for a number of data obtained by formula $1,537<a<1,627$

The cumulative distribution function for TKK obtained by formula (1) is shown in Figure 2. The cumulative distribution function for TKK obtained by formula (2) is not given this time.

The TKK obtained by formula (2) has smaller values and smaller variation than the TKK obtained by formula (1). However, this could be expected, because according to the monograph [1] presenting data concerning construction and road machinery, a very similar pattern is observed.

Taking into account our own experience, in the case being under consideration, it is worth focusing on the TKK values given by formula (1). At the same time, formula (2) is not recommended to be excluded from the express diagnostics system, as in some other cases it is highly likely to produce more accurate results.

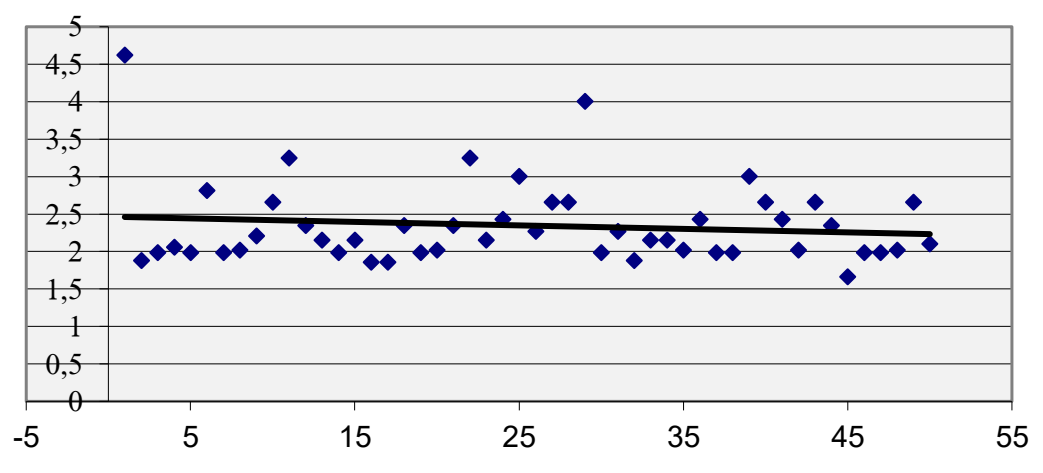

Fig. 2. The TKK cumulative distribution function for fillet weld (Formula (1)) \{confidence interval is not shown $\}$

At the end of the section the we should emphasize that both differences in the values of the TKK among different authors and some deviation from these values for the metal structures of dragline excavators further emphasize the great necessity to continue carrying out research in the sphere of issues formulated in this work. 


\section{Conclusion}

Evaluation of TKK value for fillet welds of dragline excavator boom under operating conditions can be carried out by various formulas, for example, by Afanasiev and Nikolaev formulas.

At today 's level of our knowledge and taking into account that the cycle of our research has not yet been completed, it is possible to recommend to focus on the Afanasiev formula using the system of express diagnostics to evaluate the draglain excavator condition (first of all we mean fillet welds of dragline excavator boom EH-10/70A). At the same time, the formula of Nikolayev cannot be excluded from the system of express diagnostics.

It is also useful to remember that the use of automatic welding allows to obtain more stable dimensions of welds along their length, less variation and lower values of TKK compared to TKK of welds obtained by manual welding.

In the future we plan to continue carrying out research in this field with consideration of both other formulas and other types of welds and boom types (with a significant increase in sample size and the application of more accurate estimation methods, linking them to resource assessment), which will undoubtedly correct the results obtained today.

We also plan in the future - when obtaining samples of larger volume - to divide all the main ratios of geometric dimensions into groups, which is due to the large dispersion of geometric parameters of the weld.

Generally speaking, the TKK reduction for fillet welds can be obtained due to the increase of radius of transition to base metal and the establishment of optimal ratio of thickness of base metal and thickness of welded metal.

The research carried out showed the fundamental possibility of establishing TKK, the application of which in the system of express diagnostics will allow to increase reliability of metal structures; and further study of the influence of welding methods and modes on the distribution pattern of TKK will allow to select the optimal ones, which will ensure the reduction of value and their dispersion area.

It should be noted that this research can also be of great importance for designers of dragline excavators, as TKK significantly influence calculations of metal structures [1 - 3, 6 -8 , etc.].

In our work (9) one can find other studies carried out within this framework on the subject under consideration.

\section{References}

1. G.N. Moshkaryov, V.A. Ryakhin, Durability and stability of welded structures of construction and road machines (M.: Mechanical engineering,_1984)

2. N.N. Afanasiev, Statistical theory of fatigue strength of metals (Kiev, Ukrainian Academy of Sciences, 1953)

3. Eds. G.A. Nikolayev, ete.al., -Welding in Mechanical Engineering: Reference Book. In 4 vol. (M.: Mechanical Engineering,_1979)-vol. 3 eds. V.A. Vinokurov (1979)

4. E.S. Ventzel, Probability Theory (M.: Science, 1969)

5. M.N. Stepnov, Probabilistic methods of estimation of characteristics of mechanical properties of materials and bearing capacity of structural elements (Novosibirsk: Science, 2005)

6. V.P Kogayev, Calculations for strength at stresses variable in time (M.: Mechanical engineering, 1977)

7. R.M. Schneiderovich, Strength at static and repeated-static loads (M.: Mechanical engineering, 1968) 
8. A.S. Leikin, Tension and endurance of details of complex configuration (M.: Mechanical engineering, 1968)

9. P.A. Pobegailo, D.Yu. Kritsky, GIAB, -S.50, 216 - 229 (2018) doi: 10.25018/02361493-2018-12-50-216-229 